\title{
QPSK regeneration without active phase-locking
}

Kjøller, Niels-Kristian; Da Ros, Francesco; Røge, Kasper Meldgaard; Galili, Michael; Oxenløwe, Leif Katsuo

Published in:

2016 Conference on Lasers and Electro-Optics

Publication date:

2016

Document Version

Peer reviewed version

Link back to DTU Orbit

Citation (APA):

Kjøller, N-K., Da Ros, F., Røge, K. M., Galili, M., \& Oxenløwe, L. K. (2016). QPSK regeneration without active phase-locking. In 2016 Conference on Lasers and Electro-Optics Optical Society of America (OSA).

\section{General rights}

Copyright and moral rights for the publications made accessible in the public portal are retained by the authors and/or other copyright owners and it is a condition of accessing publications that users recognise and abide by the legal requirements associated with these rights.

- Users may download and print one copy of any publication from the public portal for the purpose of private study or research.

- You may not further distribute the material or use it for any profit-making activity or commercial gain

- You may freely distribute the URL identifying the publication in the public portal

If you believe that this document breaches copyright please contact us providing details, and we will remove access to the work immediately and investigate your claim 


\title{
QPSK Regeneration without Active Phase-Locking
}

\author{
Niels-Kristian Kjøller*, Francesco Da Ros, Kasper Meldgaard Røge, Michael Galili, and Leif K. Oxenløwe \\ DTU Fotonik, Technical University of Denmark. DK-2800 Kgs. Lyngby, Denmark \\ *niekjol@fotonik.dtu.dk
}

\begin{abstract}
QPSK regeneration without active phase stabilization is investigated in numerical simulations. We propose an improved scheme for phase-locking free QPSK regeneration showing significant improvements in the error vector magnitude of the signal.

OCIS codes: (070.4340) Nonlinear optical signal processing; (060.0060) Fiber optics and optical communications;
\end{abstract}

\section{Introduction}

Phase sensitive amplification has proved promising for all-optical 'black-box' style regeneration, for the common phase-modulated data formats binary phase-shift keying (BPSK) and quadrature phase-shift keying (QPSK) [1,2], though a stable phase relation is required between the involved waves. In [3] we demonstrated a phase-locking free phase sensitive amplifier (PSA) based BPSK regenerator. Recently, we scaled it to QPSK by generating the required phase stable pump-signal set suitable for a 4-level PSA [4]. In this paper we examine numerically the performance for QPSK and propose an improved scheme comprising two 2-level PSA based regenerators operated in parallel. We find that a large performance improvement can be achieved compared to the single 4-level PSA based regenerator.

\section{Regeneration scheme}

In Fig. 1(a) a stable phase relation is maintained by a passive loop configuration in which the signal and pumps are pre-conditioned for phase sensitive amplification. This is achieved by using a delay interferometer (DI) to demodulate the QPSK signal to a 4-level amplitude modulated signal and subsequently cross-phase modulation (XPM) to modulate the signal onto a locally generated carrier recovering the QPSK modulation. See [4] for details.

(a)
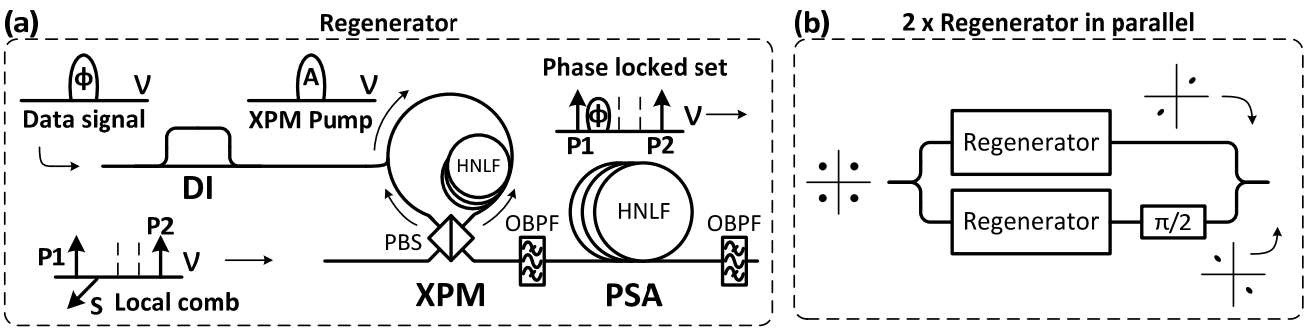

Fig. 1. (a) The phase-locking free scheme for QPSK regeneration. (b) The scheme for QPSK regeneration based on two parallel regenerators, each operated as 2-level PSAs. PBS: Polarizing beam splitter. HNLF: Highly nonlinear fiber. OBPF: Optical band-pass filter. $\pi / 2$ : Phase shift.

Fig. 1(b) illustrates dual operation in parallel. The DIs now demodulates the QPSK signal to two 2-level amplitude modulated signals each carrying half of the original information. Through XPM, two BPSK signals are recovered and regenerated in the suitably adjusted PSAs. Introducing a $\pi / 2$ phase shift in one arm, the two regenerated signals are recombined to obtain a regenerated QPSK signal.

\section{Regenerator performance}

Performance for an ideal implementation of the single and dual PSA schemes were investigated numerically using analytical transfer functions for the DI, XPM and PSA stages. A QPSK signal is generated from two dissimilar pseudorandom bit sequences (PRBSs) and each symbol represented as a phasor $a e^{i \varphi}$ degraded by a random phase error $\delta \varphi$ and propagated analytically through the system. $\delta \varphi$ is drawn from a Gaussian distribution for noise levels corresponding to different modulation indices, here defined as $m=\sqrt{2} \pi V_{R M S} / V_{\pi}$ where $V_{R M S}$ is the root-meansquare of the applied voltage and $V_{\pi}$ the half-wave voltage of the phase modulator. For the 4-level PSA we assume the optimum phase harmonic weight of 0.38 (see [5]) while for the 2-level PSAs we assume a gain factor of $g=2$.

Fig. 2(a) illustrates the constellation at different stages for the two regenerator schemes for $m=0.1$. In the top left corner the common noisy input is displayed. In the bottom row, the dual 2-level PSA scheme is investigated. The two BPSK signals are displayed in black and blue with a $\pi / 2$ relative phase shift for simplicity of illustration. Due to mixing of consecutive symbols in the DIs, each symbol carry the combined noise from two consecutive symbols as is evident at the XPM output constellations (bottom left) by the widened distributions. After the PSAs we see clear squeezing of the phase noise distributions (bottom center) while the recombination results in the 
recovery of a QPSK signal (bottom right). Besides regeneration, the squeezing in the PSAs and the recombination are seen to result in a partial conversion of phase noise to amplitude noise. In the top row of Fig. 2(a) the 4-level PSA scheme is investigated. Mixing of consecutive symbols in the DI results in broader phase noise distributions after the DI. However, the output power of the DI is limited between zero and the input power, and a squeezing occurs of the highest and lowest of the four amplitude levels close to the cut-off values. As a result we see that the noise distributions of the two symbols corresponding to the two middle amplitude levels display significantly more phase noise than the two exterior symbols after XPM (top centre). After the PSA, we clearly see the squeezing of the four levels (top right). However the two symbols with broad phase noise distributions maintain long tails though the noise is now mostly converted to amplitude noise. For $m=0.1$ the two symbols are now noisier than any of the input symbols.

(a)

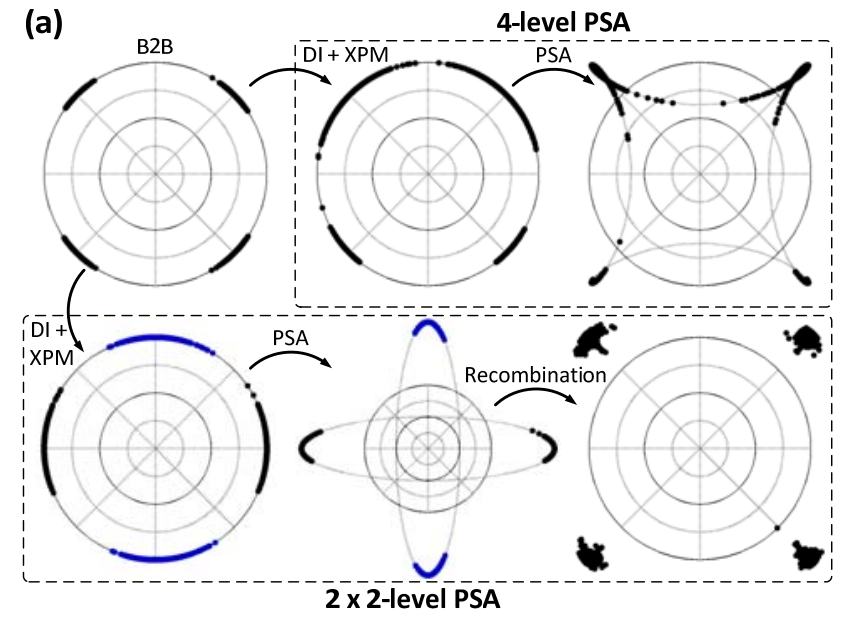

(b)

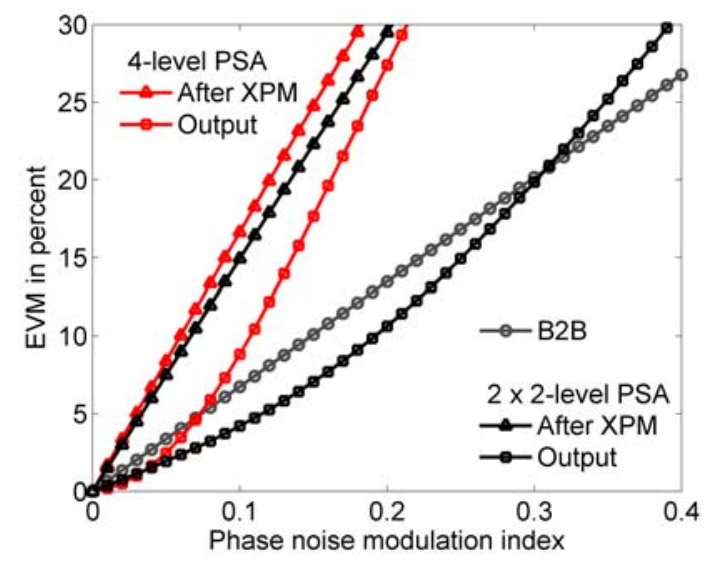

Fig. 2. Simulated regenerator performance in the presence of Gaussian phase noise for the two considered schemes. (a) Constellation diagrams at various stages for a modulation index of $m=0.10$. (b) Optical error vector magnitude (EVM) as a function of modulation index $m$.

Fig. 2(b) shows the optical error vector magnitude (EVM) [6] as a function of $m$ for the schemes, B2B, after XPM and at the output. Compared to the B2B, the XPM outputs show a clear decrease in performance due to mixing of consecutive symbols in the DIs. Regeneration in the 4-level PSA partially compensates for this degradation but it is clear from the figure that the output signal is only improved compared to the B2B for a region below $m=0.1$. In contrast, the dual 2-level PSA scheme shows improvements in EVM for values of $m$ up to around 3.3 despite the reduced EVM after XPM. The proposed dual 2-level PSA scheme thus represents a large performance improvement over the 4-level PSA based regenerator. Contributing to this improvement is primarily the even distribution of phase noise among the four symbols after XPM and the generally higher levels of phase squeezing achievable in 2-level PSAs. Note that the 4-level PSA is assumed to achieve the optimum phase harmonic weight of 0.38 (see [6]), while the 2-level PSAs are assumed to achieve more realistic sub-optimum performance with gain factors of $g=2$. Higher levels of regeneration may thus be achieved, expanding the region of $m$ values that can be regenerated, by increasing $g$.

\section{Conclusion}

The performance of the phase-locking free phase sensitive amplifier based QPSK regenerator was examined in the presence of Gaussian phase noise. We proposed an improved QPSK regenerator scheme composed of two phaselocking free regenerators operated in parallel and compared the performances. The dual regenerator scheme shows significant improvement in error vector magnitude and the ability to regenerate at several times higher noise levels.

\section{Acknowledgement}

This work is supported by the Danish Council for Independent Research (FTP) grant no. 12-127224.

\section{References}

[1] R. Slavík et al., Nature Photon. 4, 690-695, (2010).

[2] J. Kakande et al., in European Conference on Optical Communications 2010, (Torino, Italy 2010), paper PDP3.3.

[3] N. K. Kjøller et al., in Optical Fiber Communication Conference, (Los Angeles, CA, USA, 2015), paper W4C.2.

[4] N. K. Kjøller et al., in IEEE Photonics Conference 2015, (Reston, VA, USA, 2015) paper MG1.4.

[5] Stefan Wabnitz and Benjamin J. Eggleton, Springer Series in Optical Sciences vol. 194 (Springer, 2015), pp. 134-138.

[6] R. Schmogrow et al., IEEE Photon. Technol. Lett. 24, no. 1, 61-63 (2012). 